\title{
Predictive tolerance bands for the correction-less assembly of optical systems
}

Schindlbeck, Christopher, Pape, Christian, Reithmeier, Eduard

Christopher Schindlbeck, Christian Pape, Eduard Reithmeier, "Predictive tolerance bands for the correction-less assembly of optical systems," Proc. SPIE 11103, Optical Modeling and System Alignment, 111030B (30 August 2019); doi: 10.1117/12.2527659

Event: SPIE Optical Engineering + Applications, 2019, San Diego, California, United States 


\title{
Predictive tolerance bands for the correction-less assembly of optical systems
}

\author{
Christopher Schindlbeck ${ }^{a}$, Christian Pape ${ }^{a}$, and Eduard Reithmeier ${ }^{a}$ \\ ${ }^{a}$ Leibniz Universität Hannover, Institute of Measurement and Automatic Control, \\ Nienburgerstr. 17, Hanover, Germany
}

\begin{abstract}
When assembling optical systems, uncertainties of the positioning system and overall mounting tolerances lead to the deterioration of performance due to resulting misaligned optical components. In this paper, we present a novel methodology for the correction-less assembly of optical systems based on predictive tolerance bands. By running a simulation model in parallel to the assembly process, performance predictions can be made during the assembly that take into account the uncertainties of the positioning system. Typically, optical performance can be assessed by a variety of criteria. In this paper, we utilize the Maréchal criterion based on the root mean square (RMS) error as it allows to verify if the optical system is defraction-limited. The extension with Monte Carlo methods enables the prediction of mean values and standard deviations for the chosen metric. This is done for the entire optical system yet to be assembled by integrating uncertainties of the positioning system within the simulation framework. Before assembly, a desired threshold (here the RMS value derived from the Maréchal criterion) can be specified which is predicted and monitored throughout the assembly process. For verification, we analyze a two-lens system in simulation to demonstrate our proposed framework.
\end{abstract}

Keywords: Automated alignment, assembly, tolerancing, wavefronts, wavefront sensors, adaptive optics, beam expander

\section{INTRODUCTION}

Alignment of optical systems has been an ongoing research topic for the last decades. Since all positioning systems are subject to uncertainties when placing optical components, the assembled optical system will most often not perform as desired without any alignment strategy. A variety of methods exist in literature that intend to correct the optical system by the alignment of the optical components. For this, the optical components are typically equipped with passive or active adjustment mechanisms, which are able to manipulate multiple degrees of freedom by either actuators or screws depending on the mechanism. The optical components can then be either manually fine-adjusted via heuristic approaches, ${ }^{1,2}$ look-up tables, ${ }^{3}$ or by employing automatic (also: active) alignment approaches that utilize computer-aided feedback. ${ }^{4-9}$ Typically, a wavefront sensor is employed to monitor misalignments, assess the optical quality of the system, ${ }^{10-12}$ and to provide feedback for an automated alignment. However, in general, correcting optical components is a tedious and time-consuming task. Typically, it is desirable to avoid or at least decrease the time spent on this task.

In this paper, we provide a concept to evaluate the probability of a correction-less assembly of optical systems. Such assessment can be achieved by so-called tolerance bands, which we will introduce in this paper. The goal is to predict the optical performance of an optical system while also taking the uncertainties of the positioning system into account. To accommodate such uncertainties, a statistical evaluation is necessary. Therefore, we conduct a Monte Carlo simulation to obtain the probability distribution of the wavefront error.

This paper is organized as follows. Sec. 2 provides a state-of-the-art overview on the performance metrics and criteria, which will be later used in this paper. This is necessary for the construction of tolerance bands, which are introduced in Sec. 3. Therein, the theoretical background of this novel concept is outlined. In Sec. 4, we show simulation results with a simple optical system (a beam expander) as a demonstrator. Finally, Sec. 5 concludes this paper with a summary and a brief outlook.

Further author information:

E-mail: christopher.schindlbeck@imr.uni-hannover.de, Telephone: +49 5117624420

Optical Modeling and System Alignment, edited by Mark A. Kahan, José Sasián,

Richard N. Youngworth, Proc. of SPIE Vol. 11103, 111030B - () 2019 SPIE

CCC code: $0277-786 X / 19 / \$ 21 \cdot$ doi: $10.1117 / 12.2527659$

Proc. of SPIE Vol. 11103 111030B-1 


\section{PRELIMINARIES}

Given an optical system with $n$ optical components and a sensor with output $\mathbf{z}$, an input light source then maps to the sensor output via

$$
\mathbf{z}=\mathbf{h}(\mathbf{X})
$$

where $\mathbf{h}$ is (in general) a nonlinear and surjective mapping of the Hilbert space $\mathbf{X}$ (the set of all optical component poses) to the Hilbert space $\mathbf{z}$ (high-dimensional representation of the sensor image). In this paper, we will utilize the well-known Zernike coefficients (in this paper denoted by $\mathbf{z}$ ) as concise representation of the wavefront. Each optical component $i \in\{1, \ldots, n\}$ is associated with a pose vector $\mathbf{x}_{i} \in \mathbb{R}^{n_{d}}$ where $n_{d}$ are the degrees of freedom (DOF) of each optical component. To simplify the notation, it is assumed that all optical components have the same DOF although this might not always be the case. In this paper, this special case is treated, which can be extended for the general case in a straightforward manner. The inverse problem of eq. (1) consists of finding the optical poses solely from sensor measurements $\mathbf{z}$, i.e. obtaining

$$
\mathbf{X}=\mathbf{h}^{-1}(\mathbf{z}) .
$$

This problem is in general difficult to solve as it is typically ill-conditioned. ${ }^{13}$

\subsection{Performance Metrics}

In order to assess the quality of an optical system, different metrics and criteria have been developed in literature, such as the Peak-to-Valley (PV) value, the Root-Mean-Square (RMS) error, and the Strehl ratio. Although the $\mathrm{PV}$ is a common metric to specify optical system performance, the RMS should be preferred for quantitatively measuring the optical quality instead. As for the Strehl ratio, although it is immediately available in simulation, for real optical systems it is difficult to obtain because the theoretical maximum peak intensity is not readily available. Therefore, albeit not immensely useful in practice, the Strehl ratio is invaluable in optical design. ${ }^{14}$ Hence, the RMS will be used in this paper as performance metric. The RMS ${ }^{15,16}$ measures surface roughness and can be expressed conveniently in Zernike coefficients by

$$
\Phi_{\mathrm{rms}}=\sqrt{\left\langle\Phi^{2}\right\rangle-\langle\Phi\rangle^{2}}=\sqrt{\sum_{n \geq 1, m} z_{n, m}^{2}},
$$

where $z_{n, m}$ are the Zernike coefficients corresponding to the respective Zernike polynomials and brackets indicating an average value. The summation term $n \geq 1, m$ excludes piston from the RMS calculation. The equation shows that the Zernike coefficients with the largest magnitude are the main contributors to the wavefront error and are therefore the main degraders of system performance. Hence, a reduction of the Zernike coefficients directly improves the system's performance.

\subsection{Performance Criteria}

The most common optical criteria derived from the RMS to assess optical quality are

- Rayleigh (wavefront) criterion*17

The best-known benchmark for optical quality is the Rayleigh criterion. It states that an optical system with a wavefront deformation of $\left|\Phi_{\mathrm{pv}}\right|<\lambda / 4$ is perceived to be perfect. This criterion has been widely accepted as representing a minimum standard for high-quality optical performance. Since it is based on the $\mathrm{PV}$, this criterion is rather conservative.

- Maréchal criterion ${ }^{18}$

The Maréchal criterion states that a system is regarded as well-corrected if the normalized intensity at diffraction focus is greater than or equal to 0.8. This corresponds to a Strehl ratio of $S<0.80$ and to an RMS wavefront error of $\Phi_{\text {rms }} \leq \lambda / 14$ (in case of defocus). The optical system is then said to be diffractionlimited. ${ }^{19}$ As it is based on the RMS, it is more useful than the PV-based criterion of Rayleigh and can be seen as a generalization for all wavefront shapes.

\footnotetext{
${ }^{*}$ This should not be confused with the Rayleigh limit for point-image resolution.
} 
Many more performance criteria exist (such as the Danjon-Couder criterion ${ }^{20}$ ) and the interested reader is referred to literature for an exhaustive list. $^{21-23}$

\section{TOLERANCE BANDS}

So far, the statistical nature of positioning optical components has not been exploited yet for the assembly process in current state-of-the-art. In this section, the concept of tolerance bands is presented, which is a statistical construct that aims at answering the following questions:

(I) What is the probability $\alpha$ that the (remaining) optical system can be assembled by a positioning system with uncertainties $\boldsymbol{\Sigma}_{x}$ without any need for corrections?

(II) What are the maximum permissible positioning uncertainties $\boldsymbol{\Sigma}_{x, \max }$ such that the (remaining) optical system can be assembled without corrections with a certain given probability $\alpha$ ?

Now, problems (I) and (II) are addressed and corresponding strategies outlined to tackle these.

(I) Let $X$ now be a random variable of optical component poses that is normally distributed with zero mean, i.e. $X \sim \mathcal{N}\left(\mathbf{0}, \boldsymbol{\Sigma}_{x}\right)$, where $\boldsymbol{\Sigma}_{x}$ is a given covariance matrix. The goal is now to find the probability $\alpha$ such that the optical system can be assembled without the need for any corrective measures. The following steps can be made to find $\alpha$ :

(a) First, a Monte Carlo simulation is run to statistically characterize the distribution of the RMS. This is done by repeated evaluation of $\Phi_{\mathrm{rms}}$, see eq. (3). Each evaluation requires one optical simulation step $\mathbf{h}: \mathbf{X}_{i} \rightarrow \mathbf{z}$ per iteration for the computation of the RMS. Therein, $X_{i}$ is the set of optical component poses for the configuration $i$. A configuration is defined as the current step in an sequential optical system assembly and basically corresponds to the number of optical components placed in the optical train. The Monte Carlo simulation then yields a data set of samples which are distributed according to an unknown probability distribution $P$. Due to the nonlinear nature of the mapping $\mathbf{h}$ and the nonlinear and unilateral RMS metric, the RMS does not follow a normal distribution.

(b) Then, the best fit for the unknown probability distribution function (PDF) $P$ needs to be found. This can be done by fitting a variety of (common) distributions and choosing the one with the smallest fitting error.

(c) Optionally, a Kolmogorov-Smirnov test can be run to find the "goodness of fit". ${ }^{24}$

(d) In the final step, the cumulative distribution function (CDF) F needs to be computed. This allows to find the probability $\alpha$ of a (remaining) correction-less assembly by evaluating the CDF at the given RMS tolerance specification via

$$
F_{\Phi_{\mathrm{rms}}}(\mathrm{TOL})=P\left(\Phi_{\mathrm{rms}} \leq \mathrm{TOL}\right)=\alpha .
$$

These steps are repeated for each configuration $i=0, \ldots, n-1$ before or during the assembly. This enables the construction of a tolerance band for the entire assembly process. In this paper, a tolerance band is defined as the region of a chosen wavefront error metric (here RMS) between zero and a specified tolerance (here $\Phi_{\mathrm{rms}} \leq \lambda / 14$ according to the Maréchal criterion, see Sec. 1) for each step of the assembly process. Alternatively, a tolerance band (distributed symmetrically) around zero is obtained for non-unilateral metrics such as the PV. Fig. 1 shows typical results by following such an approach. Therein, a distribution of the RMS along with a PDF fit can be seen for a single configuration, see Fig. 1a. By repeating the process for every configuration, a tolerance band for the entire assembly process can be constructed, see Fig. $1 \mathrm{~b}$. The computational cost to solve the problem (I) is mostly dominated by the number of Monte Carlo runs and therefore the runtime of the optical simulation. However, it should be noted that the number of Monte Carlo runs necessary in order to obtain useful results increases exponentially with the number of optical components. 


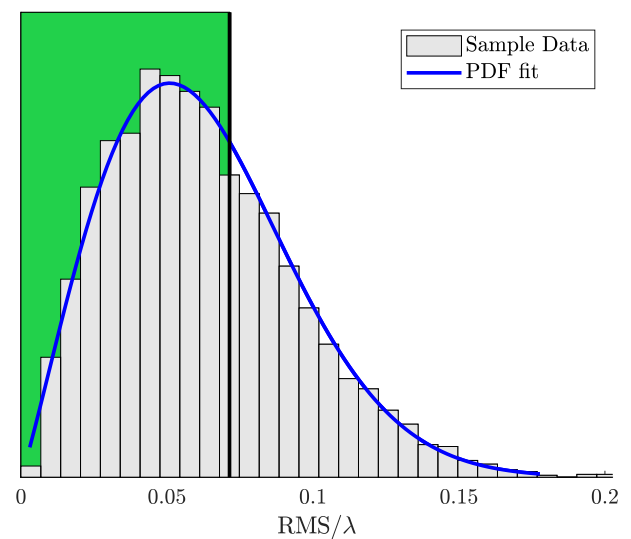

(a) Typical sample data of $\mathrm{RMS} / \lambda$ distribution and fitted PDF for a certain configuration. Green area stretches from zero to $1 / 14 \lambda \approx 0.071 \lambda$.

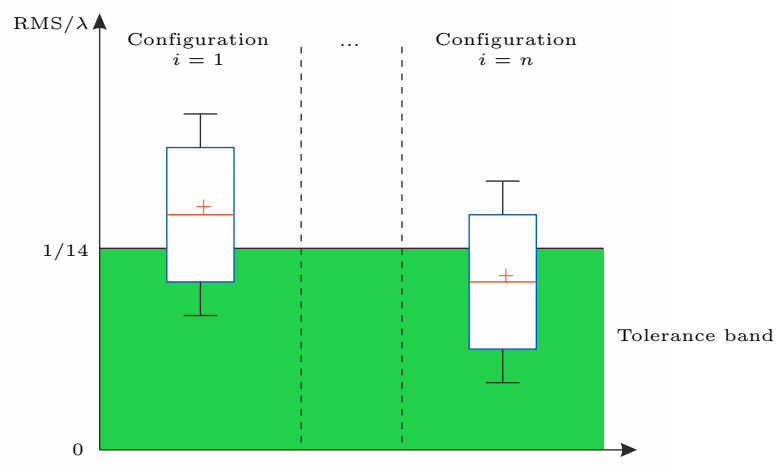

(b) Examples of box plots of sample data for every configuration and tolerance band which is in principle a concatenation of the areas seen in (a).

Figure 1: Depiction of typical results from a Monte Carlo simulation of the wavefront error. In (a), the results of a single configuration are shown, which are compactly put side-by-side with box plots in (b) to visually accommodate every configuration. Therein, the tolerance band concept becomes evident. For example, if the median lies within the green region, the (remaining) optical system can be assembled with a probability greater than or equal to $50 \%$ without any corrections.

(II) On the other hand, finding the answer to question (II) is computationally much more expensive as a stochastic optimization problem needs to be solved. In order to reduce the problem's complexity, we will only consider fully decoupled positioning uncertainties and therefore the covariance matrix has diagonal structure in this paper.

The corresponding optimization problem aims at maximizing the positioning uncertainties and is constrained by linear and stochastic equations. It is formulated as a minimization problem as follows:

$$
\begin{aligned}
\min _{\Sigma_{x}} & -\operatorname{diag}\left(\boldsymbol{\Sigma}_{x}\right)^{T} \mathbf{W}_{\Sigma} \operatorname{diag}\left(\boldsymbol{\Sigma}_{x}\right) \\
\text { subject to } & P\left(\Phi_{\mathrm{rms}} \leq \mathrm{TOL}\right)=\alpha_{d} . \\
& \operatorname{diag}\left(\boldsymbol{\Sigma}_{x}\right) \geq 0
\end{aligned}
$$

The cost function (4) aims at maximizing the permissible positioning uncertainties (eq. (6) enforces this), while the stochastic constraint (5) ensures that the optical system can be assembled with a probability greater than a given $\alpha_{d}$. The matrix $\boldsymbol{\Sigma}_{x}$ contains weights such that it is possible to prioritize positioning uncertainties in certain directions. This is the inverse problem of problem (I) and therefore many difficulties arise. Since there is no closed-form solution of eq. (2), there is also no closed-form solution to the generalized problem (4)-(6). Therefore, gradient-free optimization methods need to be employed and each function evaluation equals to an entire Monte Carlo simulation. In this paper, we will use an optimizer based on sequential quadratic programming ${ }^{25}$ in order to solve the optimization problem. Due to the underlying stochastic nature, it is much more computationally expensive to find the maximum permissible covariance matrix to a given probability. Furthermore, in general, the number of entries in the covariance matrix scale quadratically with increasing degrees of freedom for the optical component poses which in turn increases the difficulty of finding a global optimum. Therefore, this problem might even become computationally intractable for a greater number of optical components.

\section{SIMULATION RESULTS}

A beam expander with two bi-convex lenses is used as simple optical system to demonstrate the presented tolerance band concept. Fig. 2 shows a shaded 3D model of the (aligned) beam expander used in the upcoming 


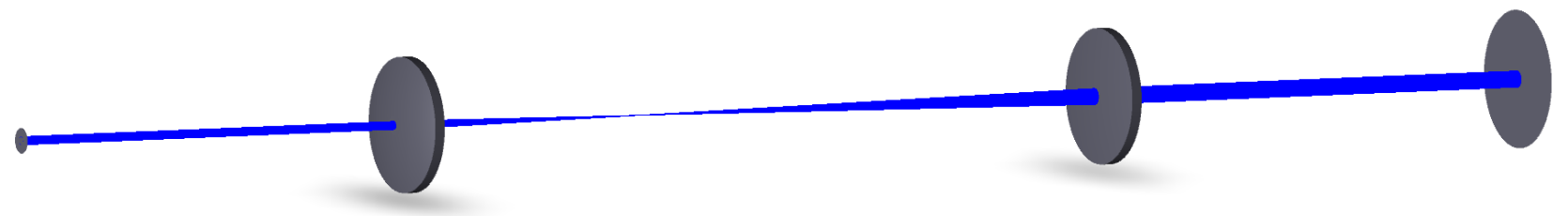

Figure 2: Ray tracing simulation with ZEMAX of the beam expander utilized in the simulation. Light source (left) emits rays that undergo two bi-convex lenses and reach a circular sensor aperture (right).

simulations. The first lens has a focal length of $50 \mathrm{~mm}$ and the second lens has a focal length of $100 \mathrm{~mm}$ resulting in a total magnification of two. Since the lenses are symmetrical around their $z$-axis, 5 degrees of freedom (3 for translation and 2 for rotation) are sufficient to fully describe them.

(I) Forward Problem

For the forward problem, we specify the positioning uncertainties and are able to calculate the probability for a correction-less remaining assembly. Due to the optical sensitivity for the utilized bi-convex lenses in this paper, the positioning uncertainty in $z^{-}, \theta_{x^{-}}$, and $\theta_{y^{-}}$-direction is not as critical as in $x$ - and $y$-direction and therefore smaller. In this paper, we first run the Monte Carlo simulation 5000 times and then we fit 17 common distributions to the obtained data. In order to find the distribution with the best fit, the Akaike Information Criterion (AIC) and/or the Bayesian Information Criterion (BIC) ${ }^{26}$ can be evaluated for example. The distribution with the lowest AIC or BIC can then be used to compute the CDF. It is then evaluated for the given RMS tolerance threshold, here $\lambda / 14$, in order to obtain the probability of a correction-less assembly. Fig. 3 shows 4 distributions with the best fit out of 17 different tested distributions. Tab. 1 lists the corresponding AIC and BIC values for the distributions. In this case, the four listed distributions resulted in a similar goodness of fit.

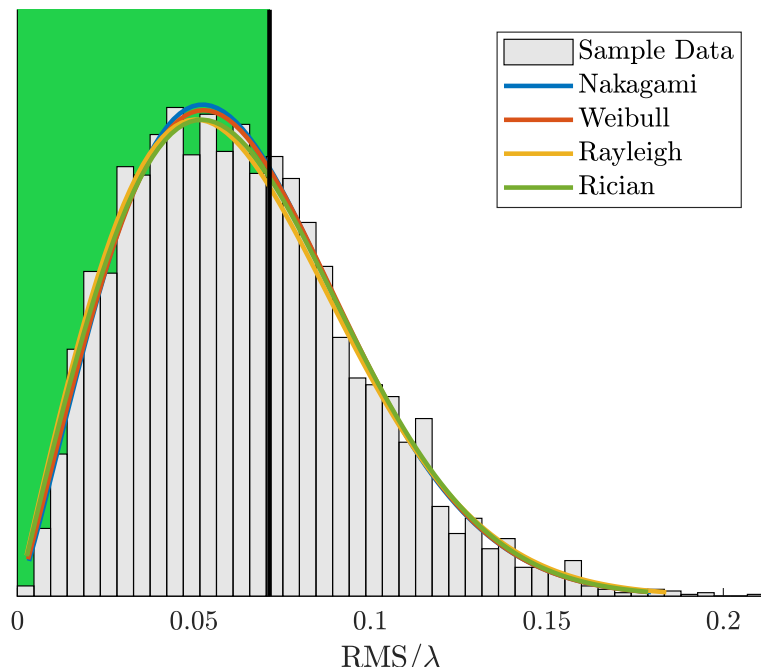

\begin{tabular}{l|cc} 
Distribution & AIC $/ 10^{-3}$ & BIC $/ 10^{-3}$ \\
\hline Nakagami & -2.0155 & -2.0158 \\
Weibull & -2.0071 & -2.0074 \\
Rayleigh & -2.0155 & -2.0158 \\
Rician & -2.0071 & -2.0074
\end{tabular}

Table 1: Finding the best distribution fit via the AIC and the BIC for Fig 3. Lower values correspond to a better fit.

Figure 3: Depiction of 4 out of 17 distributions with best fit to obtained sample data from Monte Carlo simulation.

Tab. 2 shows different positioning uncertainties at various orders of magnitude. As one would suspect, by lowering the positioning uncertainties, the probability of correction-less assembly increases. In order to achieve a probability of $100 \%$ the standard deviation (especially in $x$ - and $y$-direction) needs to be in the nanometer range. If only one lens needs to be positioned, as it is the case for configuration 1 (the first lens is here assumed to be placed at its nominal position), then lower positioning uncertainties are able to achieve higher probabilities for the correction-less assembly of the remaining optical system (here only one lens remains). Fig. 4 depicts the corresponding tolerance bands for three different positioning uncertainties. 
Table 2: Probabilities for correction-less assembly for selected given positioning uncertainties utilizing the Nakagami distribution.

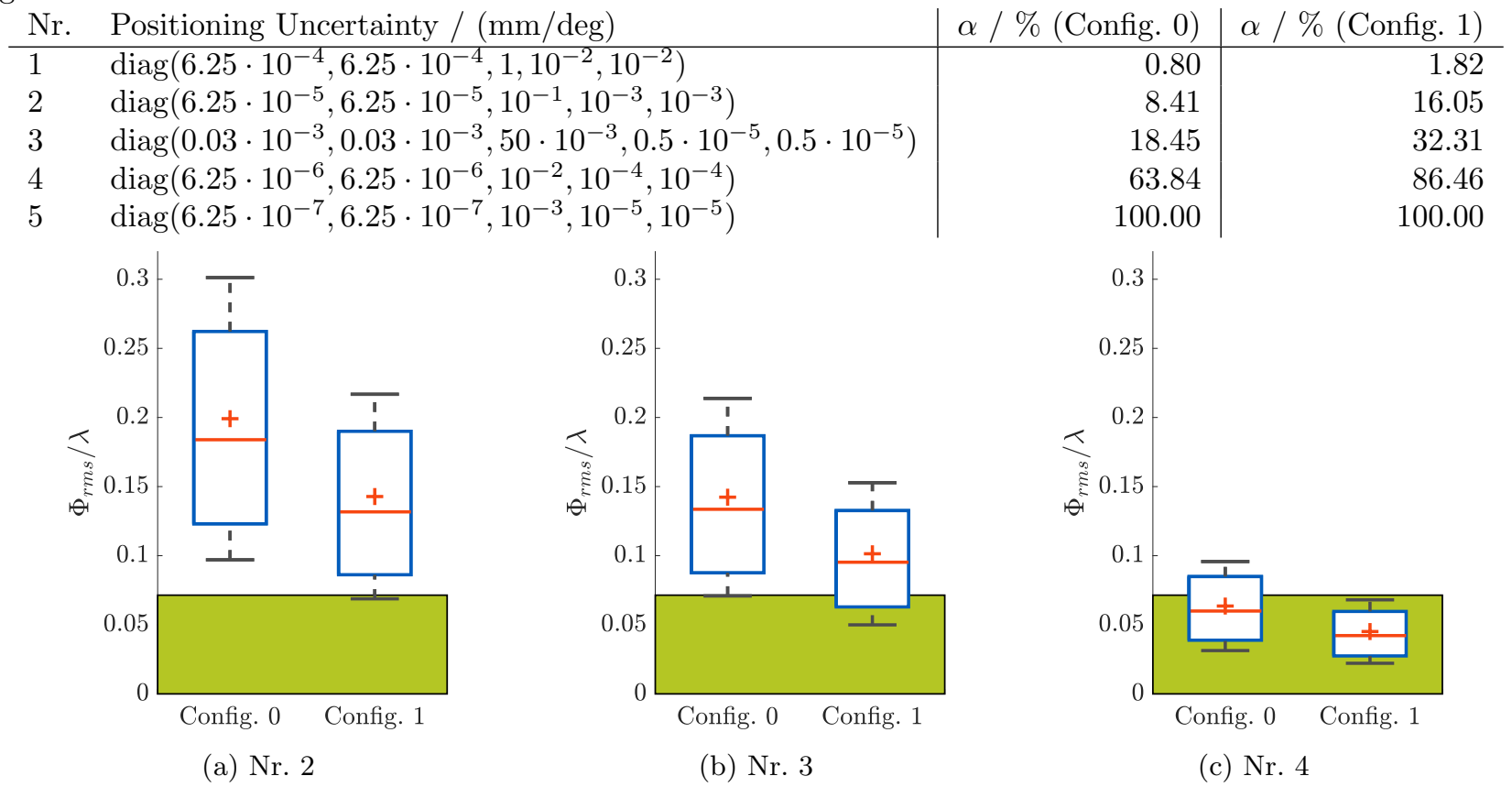

Figure 4: Tolerance bands for three different positioning uncertainties (see Tab. 2) for the simulation.

(II) Inverse Problem

Now, we solve the inverse problem, i.e. we seek the positioning uncertainties for a given probability. Tab. 3 lists a variety of probabilities for a correction-less assembly of the beam expander. Therein, the Nakagami distribution has been used to evaluate eq. (5) since it turned out to be one of the best fits for problem (I). Similarly, increasing the probability decreases positioning uncertainties. However, it should be noted

Table 3: Positioning uncertainties (right column) found by solving the proposed optimization problem given a variety of desired probabilities (left column) for configuration 0 .

\begin{tabular}{l|c}
$\alpha / \%$ & Positioning Uncertainty / $(\mathrm{mm} / \mathrm{deg})$ \\
\hline 70 & $10^{-3} \operatorname{diag}(0.0031,0.0001,2.3998,1.7548,1.9220)$ \\
80 & $10^{-4} \operatorname{diag}(0.0490,0.0253,0.3550,0.0552,0.0295)$ \\
90 & $10^{-4} \operatorname{diag}(0.0010,0.0010,0.0010,0.0010,0.0419)$ \\
99 & $10^{-5} \operatorname{diag}(0.3870,0.0790,0.0790,0.3331,0.0790)$
\end{tabular}

that the optimization problem (4)-(6) is very prone to local minima. The results are therefore not globally optimal and could be improved iteratively. For this, prior knowledge might help to adjust the weights accordingly depending on the sensitivity of the optical system.

\section{CONCLUSION}

In this paper, we have presented the concept of tolerance bands for the correction-less assembly of optical systems. It allows to evaluate the probability of assembling an optical system according to tolerance specifications without the necessity to correct optical components (problem I) or to find positioning uncertainties when given a desired probability (problem II). A simple beam expander is used as demonstrator in simulation to illustrate the basic principle of the concept. Since the wavefront RMS error does not follow a Gaussian distribution, a variety of distributions have been scrutinized to find the one with the best fit. While the forward problem is computationally manageable, especially the inverse problem suffers from the curse of dimensionality and is prone to local minima. 


\section{REFERENCES}

1. R. E. Abbink, "Interferometer alignment," 2005. US Patent 6,952,266.

2. D. R. Neal and J. Mansell, "Application of shack-hartmann wavefront sensors to optical system calibration and alignment," in Proc. Workshop on AOIM, pp. 234-243, 2000.

3. H. Gilbergs, N. Wengert, K. Frenner, P. Eberhard, and W. Osten, "Inverse calculation of position and tilt errors of optical components from wavefront data," in Modeling Aspects in Optical Metrology III, 8083, p. 808314, SPIE, 2011.

4. G. Böttger, D. Weber, F. Scholz, H. Schröder, M. Schneider-Ramelow, and K.-D. Lang, "Fully automated hybrid diode laser assembly using high precision active alignment," in Components and Packaging for Laser Systems II, 9730, p. 97300E, SPIE, 2016.

5. F. Acernese, P. Amico, M. Al-Shourbagy, S. Aoudia, et al., "The virgo automatic alignment system," Classical and Quantum Gravity 23(8), p. S91, 2006.

6. D. Kalamatianos, P. E. Wellstead, J. M. Edmunds, and P. Liatsis, "Active alignment for two-beam interferometers," Review of Scientific Instruments 77(1), p. 013103, 2006.

7. M. Holters, S. Dirks, J. Stollenwerk, and P. Loosen, "Automated and model-based assembly of an anamorphic telescope," in Optical Interconnects XVIII, 10538, p. 105380U, SPIE, 2018.

8. H. Grote, G. Heinzel, A. Freise, S. Gossler, B. Willke, H. Lück, H. Ward, M. Casey, K. A. Strain, et al., "The automatic alignment system of geo 600," Classical and Quantum Gravity 19(7), p. 1849, 2002.

9. C. G. Prevost and J. Genest, "Dynamic alignment of a michelson interferometer using a position-sensitive device," in Proc. of the SPIE 49th Annual Meeting, pp. 293-304, 2004.

10. C. Schindlbeck, C. Pape, and E. Reithmeier, "Predictor-corrector framework for the sequential assembly of optical systems based on wavefront sensing," Optics Express 26, pp. 10669-10681, 2018.

11. C. Schindlbeck, C. Pape, and E. Reithmeier, "Wavefront predictions for the automated assembly of optical systems," Proc. SPIE 10815, pp. 10815-108157, 2018.

12. C. Schindlbeck, C. Pape, and E. Reithmeier, "Process-integrated state estimation of optical systems with macro-micro manipulators based on wavefront filtering," IEEE Robotics and Automation Letters , 2019.

13. C. Schindlbeck, A Predictor-Corrector Framework for the Robot-Assisted and Automated Assembly of Optical Systems. PhD thesis, 2019.

14. D. Malacara, Optical Shop Testing, vol. 59, John Wiley \& Sons, 2007.

15. H. Gross, W. Singer, and M. Totzeck, "Handbook of optical systems," Energy 17, p. 5, 2005.

16. J. Schwiegerling, Optical Specification, Fabrication, and Testing, Spie Press, 2014.

17. R. Barakat, "Rayleigh wavefront criterion," JOSA 55(5), pp. 572-573, 1965.

18. A. Maréchal, Study of the combined effects of diffraction and geometrical aberrations on the image of a luminous point, Editions of the Journal of Optical Theatrical and Instrumental Optics, 1948.

19. S. Sinzinger and J. Jahns, Microoptics, John Wiley \& Sons, 2006.

20. A. Danjon and A. Couder, "Lunettes et telescopes-theorie, conditions d'emploi, description, reglage," Editions de la Revue d'Optique Theorique et Instrumentale, 1935.

21. L. N. Thibos, X. Hong, A. Bradley, and R. A. Applegate, "Accuracy and precision of objective refraction from wavefront aberrations," Journal of Vision 4(4), pp. 9-9, 2004.

22. P. Schoneveld, K. Pesudovs, and D. J. Coster, "Predicting visual performance from optical quality metrics in keratoconus," Clinical and Experimental Optometry 92(3), pp. 289-296, 2009.

23. W. Freude, R. Schmogrow, B. Nebendahl, M. Winter, A. Josten, D. Hillerkuss, S. Koenig, J. Meyer, M. Dreschmann, M. Huebner, et al., "Quality metrics for optical signals: eye diagram, q-factor, osnr, evm and ber," in IEEE Int. Conf. on Transparent Optical Networks, pp. 1-4, 2012.

24. F. J. Massey, "The Kolmogorov-Smirnov test for goodness of fit," Journal of the American Statistical Association 46(253), pp. 68-78, 1951.

25. J. Nocedal and S. J. Wright, "Numerical optimization 2nd," 2006.

26. G. Claeskens, N. L. Hjort, et al., "Model selection and model averaging," Cambridge Books, 2008. 